Research Article

\title{
Quick Leukocyte Nucleus Segmentation in Leukocyte Counting
}

\author{
Yapin Wang and Yiping Cao $\mathbb{D}$ \\ College of Electronic Information Engineering, Sichuan University, Chengdu, Sichuan 610064, China \\ Correspondence should be addressed to Yiping Cao; ypcao@scu.edu.cn
}

Received 23 January 2019; Revised 13 May 2019; Accepted 21 May 2019; Published 11 June 2019

Academic Editor: Kemal Polat

Copyright (c) 2019 Yapin Wang and Yiping Cao. This is an open access article distributed under the Creative Commons Attribution License, which permits unrestricted use, distribution, and reproduction in any medium, provided the original work is properly cited.

\begin{abstract}
The leukocyte nucleus quick segmentation is one of the key techniques in leukocyte real-time online scanning of human blood smear. We propose a quick leukocyte nucleus segmentation method based on the component difference in RGB color space. By analyzing the captured microscopic images of the peripheral blood smears from the autoscanning microscope, it is found that the difference values between $B$ component and $G$ component ( $B-G$ values) in the regions of the leukocyte nuclei and the platelets are much bigger than those in the other regions, even in the regions including the stains. So, the B-G values can segment the leukocyte nuclei and the platelets with an appropriate empirical threshold because the platelets are much smaller than the leukocyte nuclei, so the leukocyte nuclei can be segmented by size filtering. Also, only an 8 bit subtraction operation is performed for the B-G values, and it can improve the leukocyte nucleus segmentation speed significantly. Experimental results show that the proposed method performs well for the five types of leukocyte segmentation with a quick speed. It is very suitable for the real-time peripheral blood smear autoscanning test application. In addition, the five types of leukocytes can be counted accurately.
\end{abstract}

\section{Introduction}

The leukocyte is a kind of blood cell which can immunize humans from diseases, resist the invader, swallow foreign bodies, and produce antibodies. Thus, it has an inseparable relationship with human health [1-3]. So, leukocyte parameters are very important for human health diagnosis in clinical medicine. Presently, manual inspection is still regarded as the main leukocyte detection method in the most hospitals $[4,5]$. With the development of computer science, image processing, and pattern recognition in recent years, research on leukocytes automatic analysis system made a great progress, which has gotten a good effect on actual medical application. Leukocyte nucleus segmentation is the most basic link in leukocyte automatic analysis system. The whole process is real-time collection and analysis. That is to say the image collection and image processing are almost at the same time. Therefore, for the leukocyte automatic analysis system, the faster the leukocyte nucleus segmentation speed is, the shorter the time consumption of the image processing will be.
In order to solve this problem, a number of image segmentation methods have been proposed, for example, Cao et al. proposed a leukocyte segmentation scheme interval-valued fuzzy sets [6], Hung et al. proposed a segmentation method based on leukocyte nucleus enhancer [7], Marzuki et al. proposed a leukocyte nucleus segmentation method by using active contour [8], Pan et al. proposed a leukocyte segmentation method by using simulated visual attention (SVA) [9], Sezgin and Sankur proposed the threshold segmentation which is used to segment leukocyte nucleus based on the S component image [10], Yang et al. proposed a method of leukocyte segmentation based on $S$ component and B component image (SAB) [11], and Otsu algorithm is used in the $S$ component to segment the leukocyte nucleus [12]. The threshold is only fit for the histogram that has a clear peak or multipeaks. When processing the image with multiobject or complex background, the threshold segmentation accuracy is sometimes not as expected. Ko et al. proposed a segmentation method combining different features and mean shift clustering algorithm based on the saliency map to segment nuclei; however, the 
cytoplasm boundaries are too weak to be precisely obtained [13]. Xing et al. proposed a learning-based framework for robust and automatic nucleus segmentation with shape preservation [14]. Madhloom et al. proposed a segmentation method by using morphological operations to extract the leukocytes from other cells and background [15]. Even though some segmentation methods perform well in accuracy, the majority of these methods have some defects to some extent, such as the rigorous condition of illumination, the complicated operation of arithmetic, the difficult confirmation of parameters, the long consumption of time, and so on. In this study, we focus on developing a quick leukocyte nucleus segmentation method in leukocyte counting which can satisfy the real-time peripheral blood smear autoscanning test. Firstly, subtraction operation is conducted between B component and G component to obtain grayscale image. It can save a large amount of computing time so as to improve the processing speed and minimize the stain disturbance in the leukocyte counting; then, the leukocyte nuclei are segmented with an empirical threshold set by lots of experimental analysis statistics; finally, the leukocyte nuclei are located accurately by using horizontal and vertical projection [16, 17].

\section{Leukocyte Nucleus Segmentation}

When using above existing segmentation methods for leukocyte nucleus segmentation, the segmentation results might be sensitively influenced by the surrounding conditions and dyeing conditions. Some methods may play higher segmentation accuracy but their segmentation speed may not be so fast to be used in real-time online leukocyte testing. Some methods play a convenient segmentation speed, but their segmentation accuracy may not be good for leukocyte nucleus segmentation. It is found that the leukocyte nuclei and platelets can be segmented by the $B-G$ values of the captured blood smear microscopic images; because the platelets are much smaller than the leukocyte nuclei, the leukocyte nuclei can be segmented by size filtering. The final segmentation can perform accurately and quickly.

2.1. Segmentation Parameter Selection. Figures 1(a)-1(c) show three typical captured leukocytes at different surrounding conditions and different dyeing conditions. Figure 1(d) shows the R, G, and B components' cutaway views on the dashed line $A A^{\prime}$ in Figure 1(a). Figure 1(e) shows the $\mathrm{R}, \mathrm{G}$, and $\mathrm{B}$ components' cutaway views on the dashed line $B B^{\prime}$ in Figure 1(b). Figure 1(f) shows the R, G, and $B$ components' cutaway views on the dashed line $C C^{\prime}$ in Figure 1(c). The bold solid line rectangle ranges in Figures 1(d)-1(f) indicate the leukocyte nucleus' regions. Figure $1(\mathrm{~g})$ shows the $\mathrm{B}-\mathrm{G}$ component's cutaway view on the dashed line $A A^{\prime}$ in Figure 1(a). Figure 1(h) shows the $\mathrm{B}-\mathrm{G}$ component's cutaway view on the dashed line $B B^{\prime}$ in Figure 1(b). Figure 1(i) shows the B-G component's cutaway view on the dashed line $C C^{\prime}$ in Figure 1(c). From Figures $1(d)-1(i)$, we can see that the $B-G$ values in the regions of the leukocyte nuclei are always much bigger than those in other regions whether the surrounding conditions and dyeing conditions change or not. So, we can use B-G values to segment the leukocyte nuclei. From Figures 1(a)1(c), we can also see that the dashed circled platelets have almost the same color as the leukocyte nuclei in the same images whether the surrounding conditions and dyeing conditions change or not, so the $B-G$ values can segment both the leukocyte nuclei and platelets at the same time; because the platelets are much smaller than the leukocyte nuclei, the leukocyte nuclei can be segmented by size filtering. It means that $B-G$ values can be selected as the parameters to segment the leukocyte nuclei robustly for different surrounding conditions and different dyeing conditions.

But actually, when preparing the blood smear, the stains are unavoidable. Figures 2(a)-2(c) show three typical captured leukocytes with stains at different surrounding conditions and different dyeing conditions. Figures 2(d)-2(f), show the R, G, and B components' cutaway views on the dashed lines in Figures 2(a)-2(c), respectively, which include the leukocyte nucleus regions (bold solid line rectangles). Figures 2(g)-2(i) show the B-G component's cutaway views on the dashed lines in Figures 2(a)-2(c), respectively, which include the leukocyte nucleus regions. Figures 2(j)2(l) show the R, G, and B components' cutaway views on the dashed dotted lines in Figures 2(a)-2(c), respectively, which include the stain regions (bold dashed line rectangles). Figures 2(m)-2(o) show the B-G component's cutaway views on the dashed dotted lines in Figures 2(a)-2(c), respectively, which include the stain regions. By comparing them, the $B-G$ values in the stain regions are alterable in a certain numerical range which are smaller than 50 concluded by lots of experimental analysis statistics while those in the regions of the leukocyte nuclei are always bigger than 110 , so we can set an appropriate empirical segmentation threshold $T_{0}\left(T_{0} \in[50,100]\right)$ to minimize the stain disturbance at different surrounding conditions and different dyeing conditions. So, the B-G values used as the segmentation parameters show their good stability and robustness.

\subsection{Algorithm Analysis for the Leukocyte Nucleus Segmen-} tation Method. Because only an 8 bit subtraction operation is performed for the $B-G$ values, it has the shortest time consumption in all existing operations such as initialization operation, float operation, and double operation. Also, the segmentation threshold need not be calculated by some algorithms such as Otsu's algorithm and mean shift clustering algorithm; it is set to be $T_{0}$ by experience through the experiments of thousands samples of leukocyte nuclei images. This method can avoid the long time consumption of searching for the segmentation threshold. Although the size filtering must be done, it is a common shared operation for all leukocyte segmentation methods. So, the total consumption time is still the shortest as well which means that the proposed method is a quick leukocyte nucleus segmentation method recently. The basic process of the leukocyte nucleus segmentation algorithm is as follows: 
A

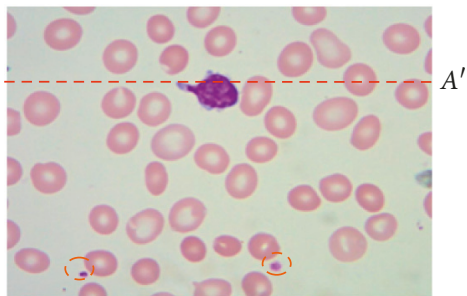

(a)

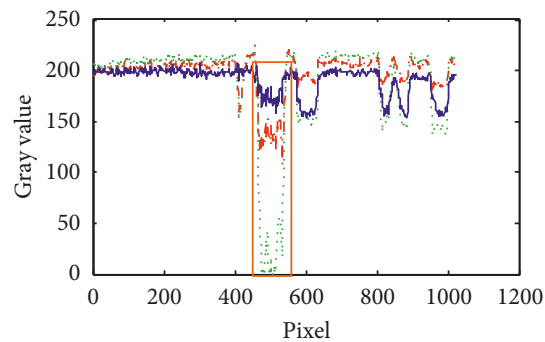

-.- R component

$\mathrm{G}$ component

- B component

(d)

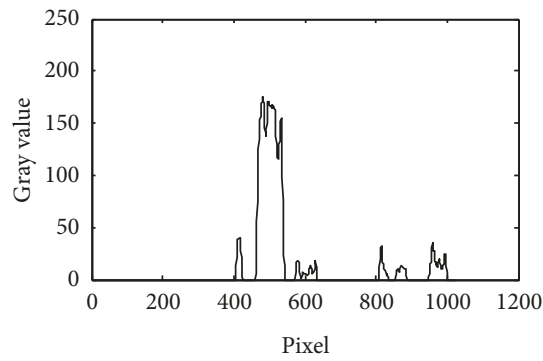

— DBG component

(g)

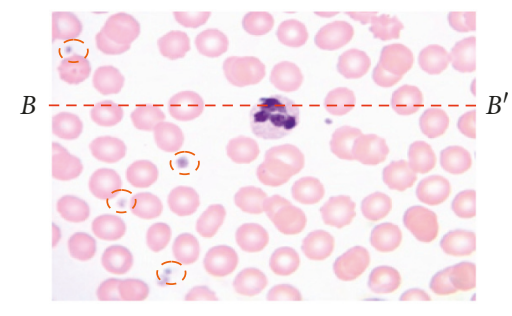

(b)

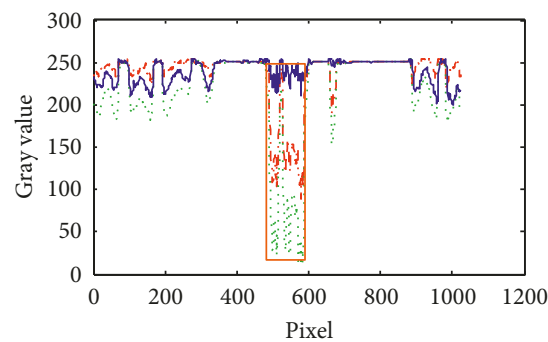

-..- R component

G component

- B component

(e)

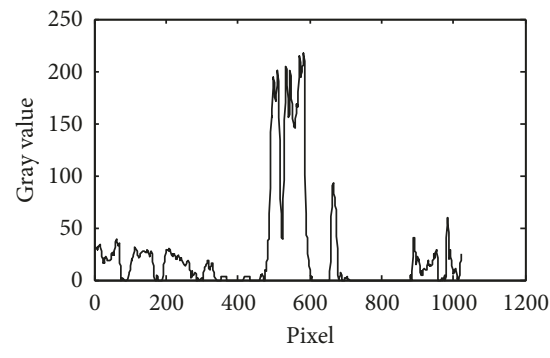

DBG component

(h)

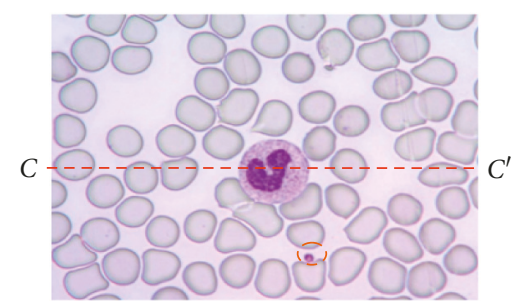

(c)

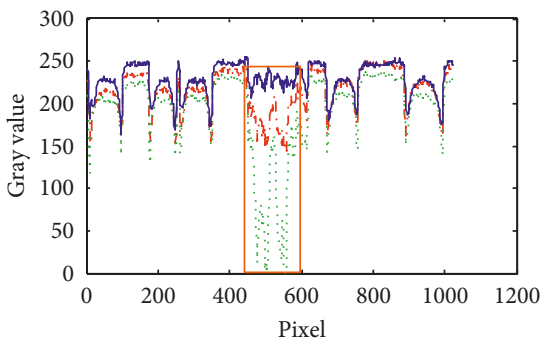

-. - R component

G component

- B component

(f)

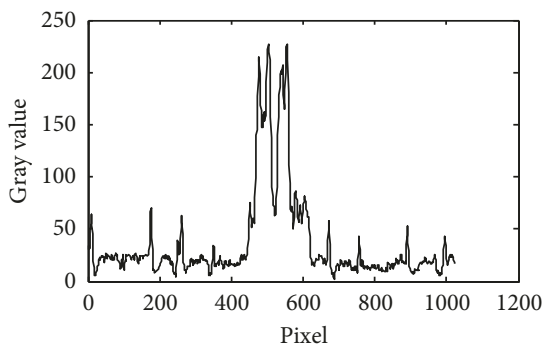

_ DBG component

(i)

FIgURE 1: The R, G, and B components' cutaway views for captured leukocyte images at different conditions. (a) The captured leukocyte image 1. (b) The captured leukocyte image 2. (c) The captured leukocyte image 3. (d) The R, G, and B components' cutaway views on $A A^{\prime}$ of (a). (e) The R, G, and B components' cutaway views on $B B^{\prime}$ of (b). (f) The R, G, and B components' cutaway views on $C C^{\prime}$ of (c). (g) The B-G component's cutaway view on $A A^{\prime}$ of (a). (h) The $B-G$ component's cutaway view on $B B^{\prime}$ of (b). (i) The $B-G$ component's cutaway view on $C C^{\prime}$ of (c).

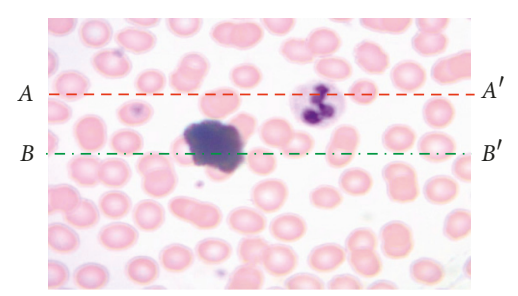

(a)

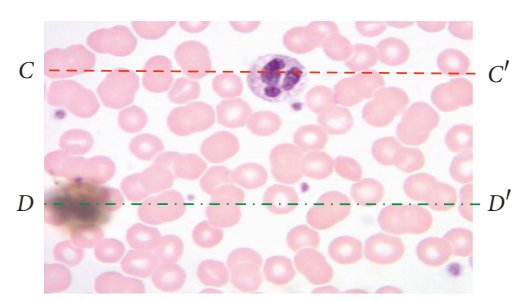

(b)

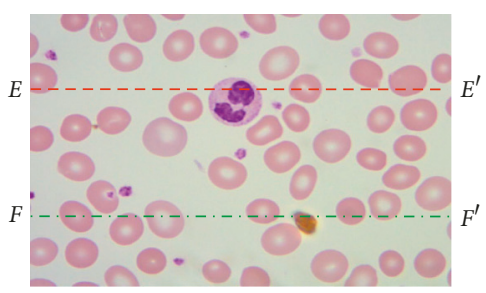

(c)

Figure 2: Continued. 


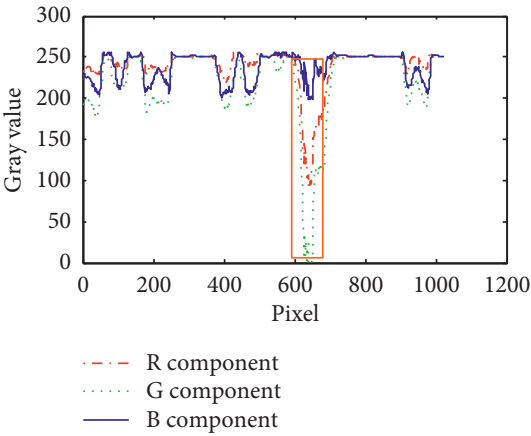

(d)

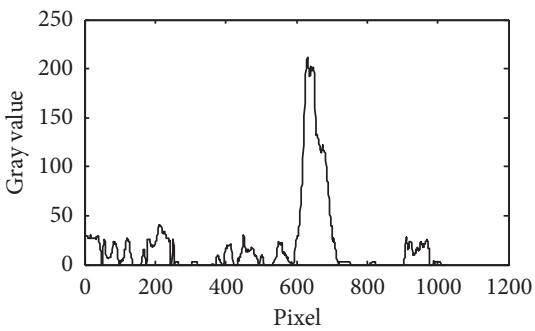

— DBG component

(g)

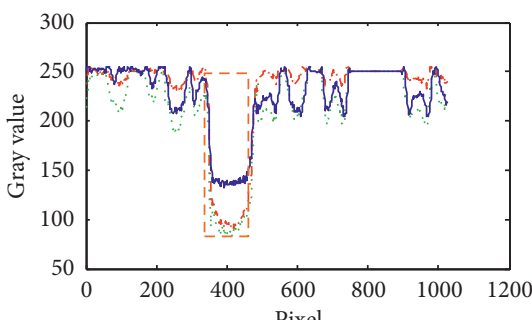

-. - R component G component — B component

(j)

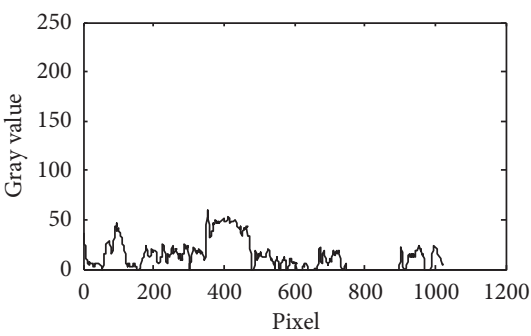

— DBG component

(m)
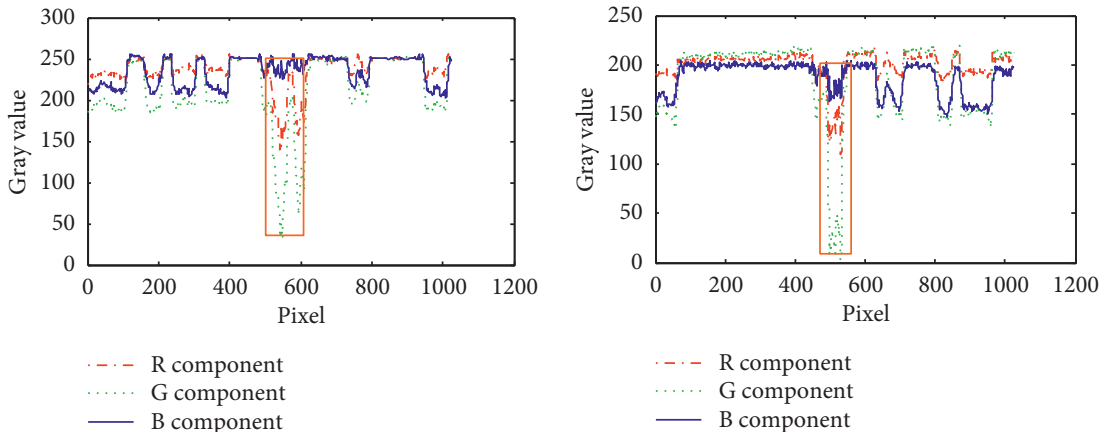
-... R component - B component

(e)

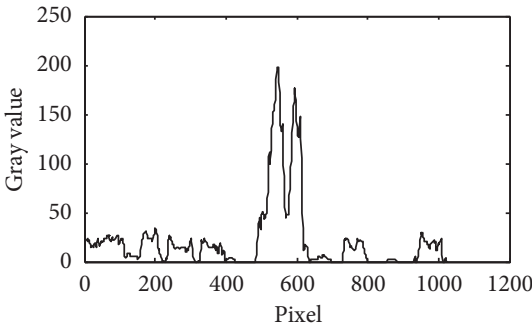

_ DBG component

(h)

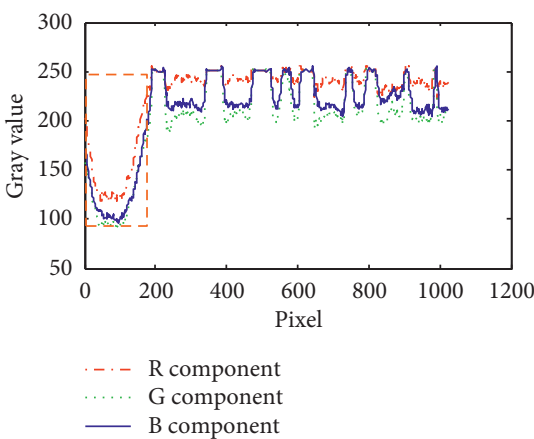

(k)

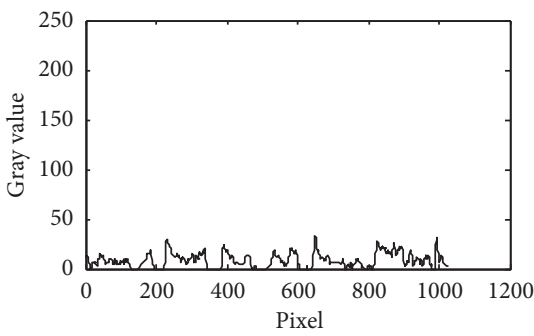

- DBG component

(n) (f)

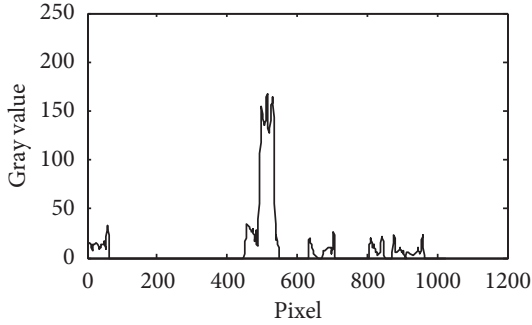

— DBG component

(i)

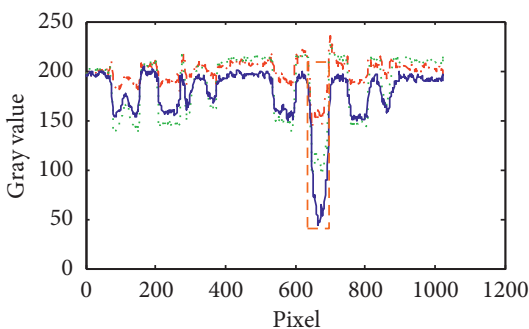

-.- R component G component - B component

(l)

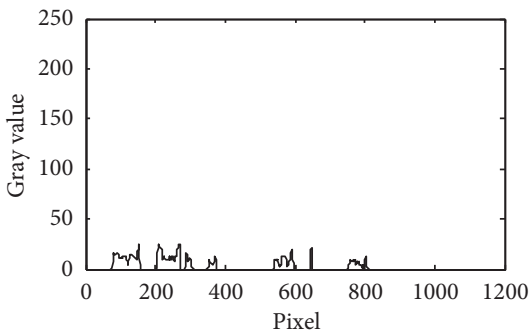

(o)

Figure 2: The R, G, and B components' cutaway views for captured leukocyte images with stains at different conditions. (a) The leukocyte captured image 1. (b) The leukocyte captured image 2. (c) The leukocyte captured image 3. (d) The R, G, and B components' cutaway views on $A A^{\prime}$ of (a). (e) The R, G, and B components' cutaway views on $C C^{\prime}$ of (b). (f) The R, G, and B components' cutaway views on $E E^{\prime}$ of (c). (g) The B - G component's cutaway view on $A A^{\prime}$ of (a). (h) The B - G component's cutaway view on $C C^{\prime}$ of (b). (i) The B-G component's cutaway view on $E E^{\prime}$ of (c). (j) The R, G, and B components' cutaway views on $B B^{\prime}$ of (a). (k) The R, G, and B components' cutaway views on $D D^{\prime}$ of (b). (l) The R, G, and B components' cutaway views on $F F^{\prime}$ of (c). (m) The B - G component's cutaway view on BB' of (a). (n) The B - G component's cutaway view on $D D^{\prime}$ of (b). (o) The B - G component's cutaway view on $F F^{\prime}$ of (c). 
(1) Extracting the B component and G component, respectively.

(2) Making the subtraction operation between the B component and $\mathrm{G}$ component to obtain the gray image.

(3) Getting the binarization image with an appropriate empirical threshold for the gray image.

(4) Eliminating the platelets by size filtering to get the segmentation result.

(5) Labeling the connected regions with different gray values for the segmentation result as shown in Figure 3; after leukocyte nucleus segmentation, if there are more than one segmented leukocyte nucleus regions in an image, the judgment of lobular nuclei will be made. Firstly, the areas of all the leukocyte nucleus regions will be calculated. The maximal statistic area value of lobular region is $S_{0}$. The segmented region whose area is larger than $S_{0}$ will not be a lobocyte. For the rest regions, the empirical value of the Euclidean distance between the centroids is adopted to judge the lobular nuclei. The Euclidean distance $D$ between the centroids of any two regions is calculated. If the distance $D$ is bigger than the distance empirical value $D_{0}$ (set by large numbers of experimental statistical), it means that the two regions are two independent leukocyte nuclei. Otherwise, they belong to the same leukocyte nucleus. The above steps are repeated until all the regions have been judged.

(6) Making the projection operation for the each labeled connected region to obtain the envelope by using the following equations:

$$
\begin{aligned}
&\left(x_{1}, x_{\mathrm{r}}\right)=\operatorname{Prj}\left\{I_{\mathrm{BGS}}\right\}, \\
&\left(y_{\mathrm{t}}, y_{\mathrm{b}}\right)=\operatorname{Prj}_{x}\left\{I_{\mathrm{BGS}}\right\}, \\
&\left\{\begin{aligned}
x_{\mathrm{c}} & =\operatorname{INT}\left\{\frac{x_{1}+x_{\mathrm{r}}}{2}\right\}, \\
y_{\mathrm{c}} & =\operatorname{INT}\left\{\frac{y_{\mathrm{t}}+y_{\mathrm{b}}}{2}\right\}, \\
w & =x_{\mathrm{r}}-x_{\mathrm{l}}, \\
h & =y_{\mathrm{t}}-y_{\mathrm{b}},
\end{aligned}\right.
\end{aligned}
$$

where the $x_{\mathrm{l}}, x_{\mathrm{r}}, y_{\mathrm{t}}$, and $y_{\mathrm{b}}$ are the leftmost boundary, rightmost boundary, topmost boundary, and bottommost boundary, respectively, $I_{\mathrm{BGS}}$ is the segmented nucleus, $\operatorname{Prj}_{y}$ \{.\} and $\operatorname{Prj}_{x}\{$.$\} are the vertical projection operator and the$ horizontal projection operator, respectively, $\left(x_{\mathfrak{c}}, y_{\mathrm{c}}\right)$ is the center of each leukocyte nucleus, the operator INT\{.\} is the meaning of integralization operator, and $w$ and $h$ are the width and the height of each leukocyte nucleus, respectively.

In case of important artifacts which are characterized by same color and size with the leukocyte nucleus, a judgment based on leukocyte feature is made to distinguish a leukocyte from an artifact after the real-time obtaining of the segmented images $[18,19]$. The ratio of areas of cytoplasm and nucleus is the selected leukocyte feature. According to lots of experimental analysis statistics, the minimum value of the ratio of areas of cytoplasm and nucleus is 0.26 for leukocyte. If the ratio of areas of cytoplasm and nucleus is smaller than 0.26 , the segmented image will be regarded as an artifact and be discarded. In order to obtain the region of cytoplasm, Yang's method using B component and saturation (S) component is adopted [11]. Figure 4 shows some of the segmented artifacts, and they are distinguished by the above mentioned method.

Thousands of leukocyte micrographic images are selected as experimental samples. Five typical different leukocytes from those samples are shown in Figure 5(a). They are described as in Rezatofighi and Soltanian-Zadeh [20]; Chan et al. [21]; and Rezatofighi and Soltanian-Zadeh [20]. They are basophil (Bas), eosinophil (Eos), neutrophil (Neu), lymphocyte (Lym), and monocyte (Mon) from left to right, respectively. The Bas has large granules which are dyed deep blue to purple and are often so numerous to mask the nucleus; the Eos has large granules which are acidophilic and appear pink (or red) after dyeing; the Neu has very tiny dyed granules with low visibility, and its nucleus is frequently multilobed with lobes connected by thin strands of nuclear material; the Lym has a very clear cytoplasm that is pale blue when dyed; the Mon is the largest among the leukocytes, and its nucleus is often " $U$ " shaped or kidney bean shaped while its cytoplasm is abundant and light blue. Figures 5(b)-5(e) show the intermediate results processed by the proposed method. We can find that the nuclei for the five types of leukocyte can be located ideally though the surrounding conditions or the dyeing conditions are different. It shows the robustness of the proposed leukocyte nucleus segmentation algorithm. If an auxiliary analysis is performed with some other characteristics of the leukocyte, the five types of leukocytes can be classified and counted effectively.

\section{Experimental Result Analysis}

All the algorithms were implemented with Visual C++ 6.0 on a Windows XP operating system with a $3.30 \mathrm{GHz}$ Intel Core i3-3220 CPU and 4GB memory. Peripheral blood smears are prepared with Wright-Giemsa dye shown in Figure 6(a). More than 3000 different microscopic images with $1024 * 768$ sizes were captured by a CCD camera, and they were collected from the People's Hospital of Sichuan Province. The experimental system is shown in Figure 6(b), an improved OLYMPUS BX53 microscope which can realize autocapturing leukocyte image with a MD55 camera, autofocusing by electrical driving the stage up and down and autoscanning by electrical driving the stage rightward and leftward or forward and backward with a three-axis step motor controlling system.

In order to analyze the segmentation accuracy and the real-time performance of the proposed segmentation method, segmentation accuracy data and time consumption data are recorded and compared with the existing method 


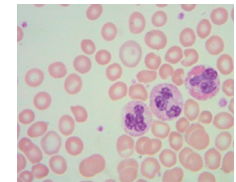

(a)

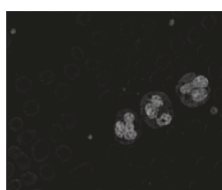

(b)

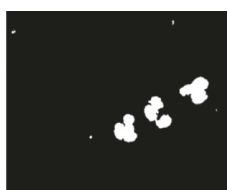

(c)

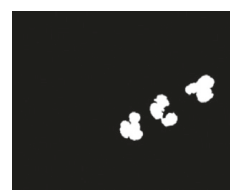

(d)

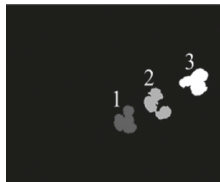

(e)

FIgURE 3: The connected region labeling image. (a) The multileukocyte captured image. (b) The B-G image. (c) The binarization image. (d) The segmented nucleus. (e) The connected region labeled image of (d).

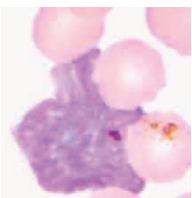

(a)

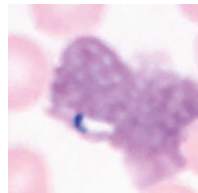

(b)

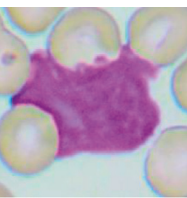

(c)

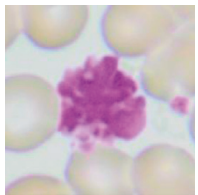

(d)

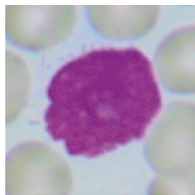

(e)

Figure 4: Some of the distinguished artifacts. (a) Artifact 1. (b) Artifact 2. (c) Artifact 3. (d) Artifact 4 . (e) Artifact 5.
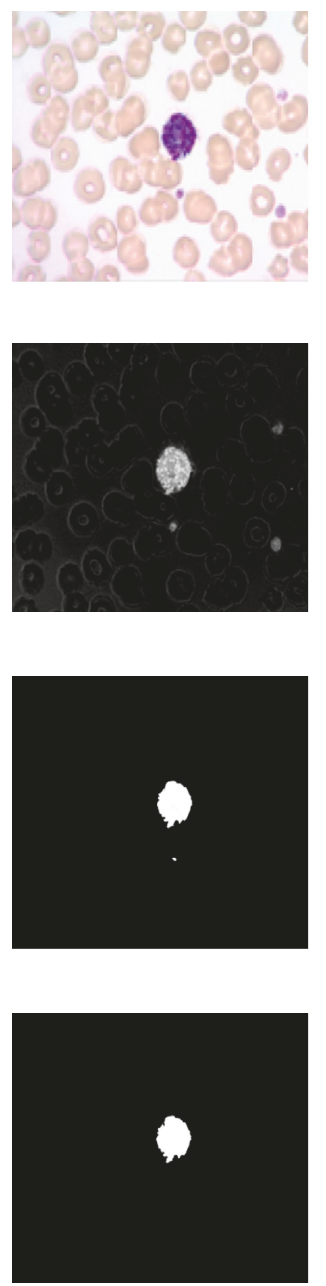
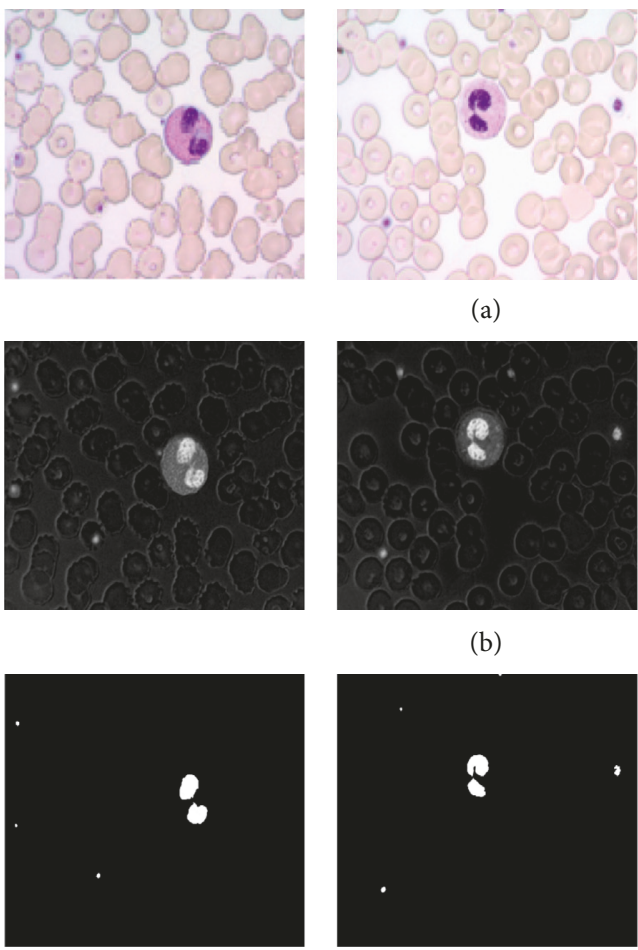

(a)

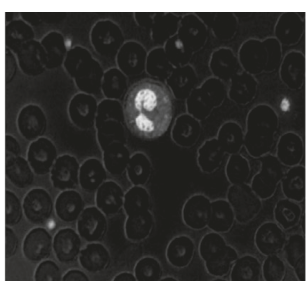

(b)

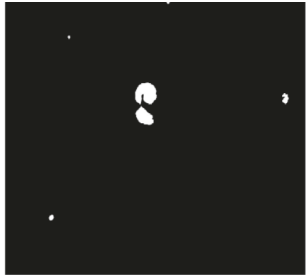

(c)
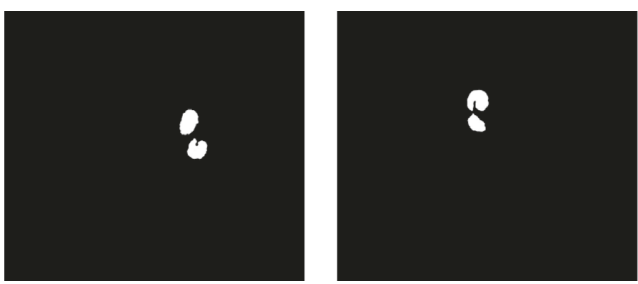

(d)
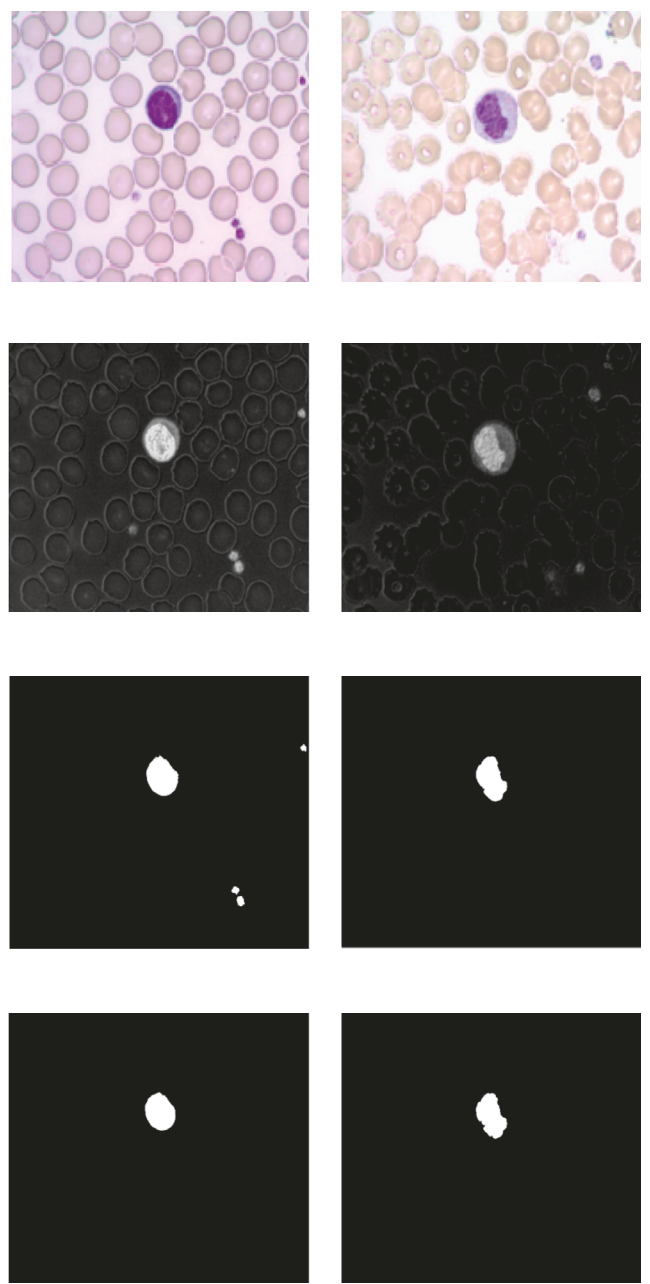

Figure 5: Continued. 

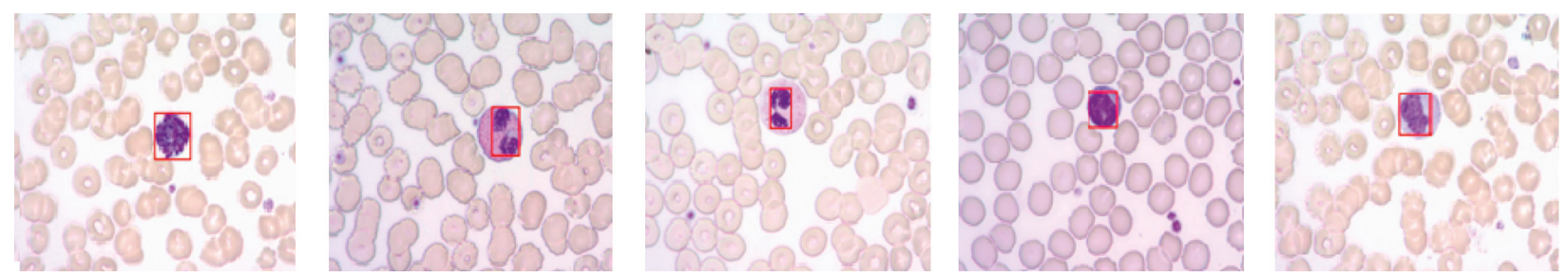

(e)

Figure 5: The segmentation process. (a) The captured leukocyte images. (b) The B-G images. (c) The binarization images. (d) The segmentation results. (e) The leukocyte nucleus location.

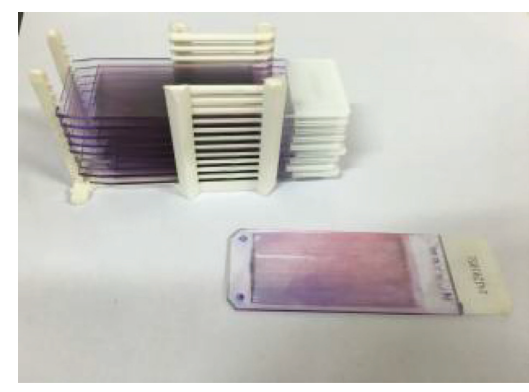

(a)

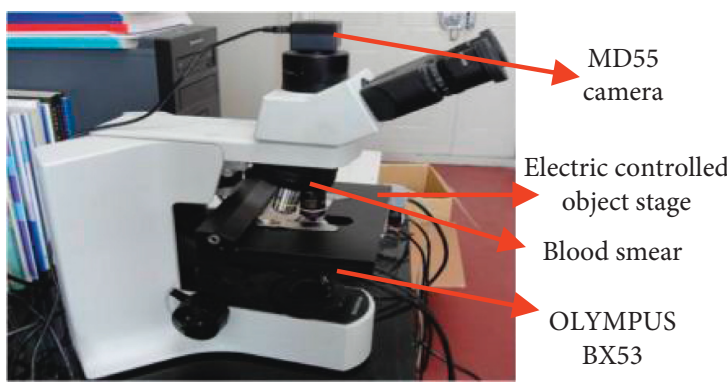

(b)

FiguRE 6: The blood smears and the autoscanning system experimental device. (a) Blood smears. (b) Autoscanning system experimental device.

SAB based on Otsu's algorithm [11] and Ko et al.'s method based on mean shift clustering [13]. The segmentation standard of the leukocyte nucleus is manually extracted by the specialist in hematology.

\subsection{Leukocyte Nucleus Segmentation Experiment Results.} The leukocyte nucleus segmentation accuracy of the three methods is shown in Table 1. Over 577 representative samples at different degrees of dyeing or different illumination conditions are chosen for analysis.

It is easy to find from the experimental results that all three methods perform well. When using the proposed method, the nucleus segmentation accuracies of the five types of leukocytes are all more than $90 \%$. In spite of the small sample, the segmentation accuracies of basophils can also reach $88.2 \%$ using the SAB and Ko's method. The proposed method performs a little worse for Eos than the $\mathrm{SAB}$, but the difference is very small. And the average segmentation accuracy of the proposed method is $97.40 \%$, which is higher than that of the SAB (95.40\%) and that of Ko's method (92.44\%). The experimental results show the stability and high accuracy of the proposed method.

3.2. Leukocyte Nucleus Segmentation Speed Analysis. The average time consumptions for the five types of leukocytes by the three nucleus segmentation methods for the above 577 samples are shown in Table 2.

We can see from Table 2 that the average time consumption for the leukocyte nucleus segmentation by the proposed method, Ko's method, and the SAB method is
$0.26 \mathrm{~ms}, 2.6 \mathrm{~ms}$, and $27.15 \mathrm{~ms}$, respectively. It reveals that the proposed method is ten times quicker than Ko's method and over one hundred times quicker than the SAB method. So, the proposed method has better timeliness to meet the real-time requirement in the blood smear autoscanning test application.

3.3. Leukocyte Autoscanning and Autocounting. The quick segmentation technique has been applied in the real-time autoscanning for the leukocyte peripheral blood smear. Thousands of blood smear samples have been scanned automatically. In order to provide sufficient information for doctor to diagnose disease efficiently, 100 leukocyte images from each blood smear must be extracted by a real-time leukocyte autoscanning system. Figure 7 shows the leukocyte autoscanning results for two of the blood smear samples at different surrounding conditions and different dyeing conditions. The leukocyte images are realistically well extracted. Table 3 shows the leukocyte counting results for ten tested blood smear samples in which sample 1 and sample 2 are the same samples shown in Figure 7. We can see from Figure 7 that the proportions of Neu, Lym, Mon, Eos, and Bas are $66 \%, 24 \%, 5 \%, 4 \%$, and $1 \%$, respectively, in sample 1 while those in sample 2 are $60 \%, 26 \%, 10 \%, 3 \%$, and $1 \%$, respectively. In this way, the corresponding proportions of different samples can be concluded accurately. Also, the average time consumption for leukocyte counting is 100 $( \pm 1)$ seconds per blood smear; most of the time is spent for the mechanical movement while scanning. The segmentation time consumption in the real-time leukocyte autoscanning system might be less than 2 milliseconds per 
TABLE 1: Segmentation accuracy of the five types of leukocytes.

\begin{tabular}{|c|c|c|c|c|c|c|c|}
\hline \multirow{3}{*}{$\begin{array}{l}\text { Types of } \\
\text { leukocyte }\end{array}$} & \multirow{3}{*}{$\begin{array}{l}\text { Numbers of } \\
\text { samples }\end{array}$} & \multicolumn{6}{|c|}{ Segmentation method } \\
\hline & & \multicolumn{2}{|c|}{$\begin{array}{c}\text { SAB } \\
\text { (based on Otsu's algorithm) }\end{array}$} & \multicolumn{2}{|c|}{$\begin{array}{c}\text { Ko's method } \\
\text { (based on mean shift clustering) }\end{array}$} & \multicolumn{2}{|c|}{ The proposed method } \\
\hline & & Result of segment & $\begin{array}{c}\text { Accuracy } \\
(\%)\end{array}$ & $\begin{array}{c}\text { Result of } \\
\text { segment }\end{array}$ & $\begin{array}{c}\text { Accuracy } \\
(\%)\end{array}$ & $\begin{array}{l}\text { Result of } \\
\text { segment }\end{array}$ & $\begin{array}{c}\text { Accuracy } \\
(\%)\end{array}$ \\
\hline Eos & 45 & 44 & 97.8 & 41 & 91.1 & 43 & 95.5 \\
\hline Bas & 17 & 15 & 88.2 & 15 & 88.2 & 16 & 94.1 \\
\hline $\mathrm{Neu}$ & 246 & 242 & 98.4 & 232 & 94.4 & 245 & 99.6 \\
\hline Mon & 138 & 133 & 96.4 & 129 & 93.8 & 136 & 98.6 \\
\hline Lym & 131 & 126 & 96.2 & 124 & 94.7 & 130 & 99.2 \\
\hline
\end{tabular}

TABle 2: Time consumptions for the leukocyte nucleus segmentation.

\begin{tabular}{|c|c|c|c|c|}
\hline \multirow[b]{2}{*}{ Types of leukocyte } & \multirow[b]{2}{*}{ Numbers of samples } & \multicolumn{3}{|c|}{ Average time consumptions for each leukocyte (ms) } \\
\hline & & $\begin{array}{c}\text { SAB } \\
\text { (based on Otsu's algorithm) }\end{array}$ & $\begin{array}{c}\text { Ko's method } \\
\text { (based on mean shift clustering) }\end{array}$ & $\begin{array}{c}\text { The proposed } \\
\text { method }\end{array}$ \\
\hline Eos & 45 & 27.83 & 2.42 & 0.26 \\
\hline Bas & 17 & 26.72 & 2.00 & 0.27 \\
\hline Neu & 246 & 27.59 & 3.51 & 0.24 \\
\hline Mon & 138 & 27.14 & 3.17 & 0.25 \\
\hline Lym & 131 & 26.45 & 1.91 & 0.26 \\
\hline \multicolumn{2}{|c|}{ Average } & 27.15 & 2.60 & 0.26 \\
\hline
\end{tabular}

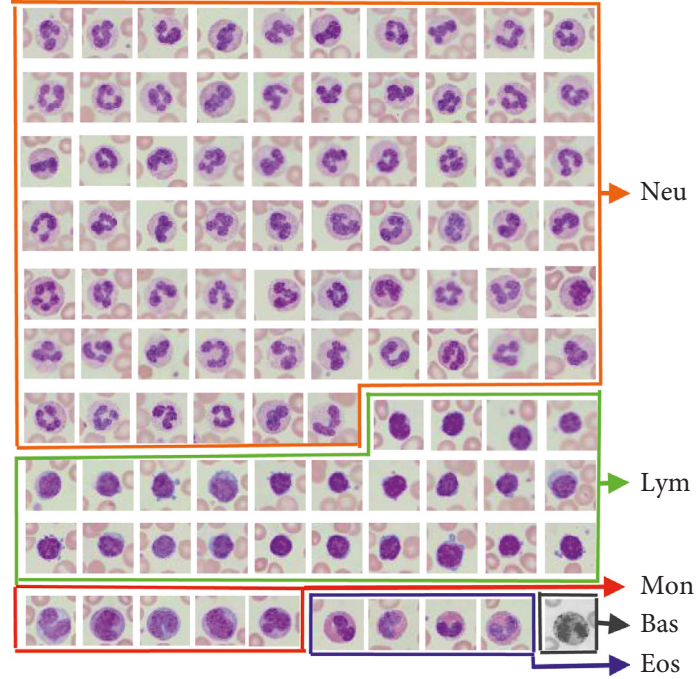

(a)

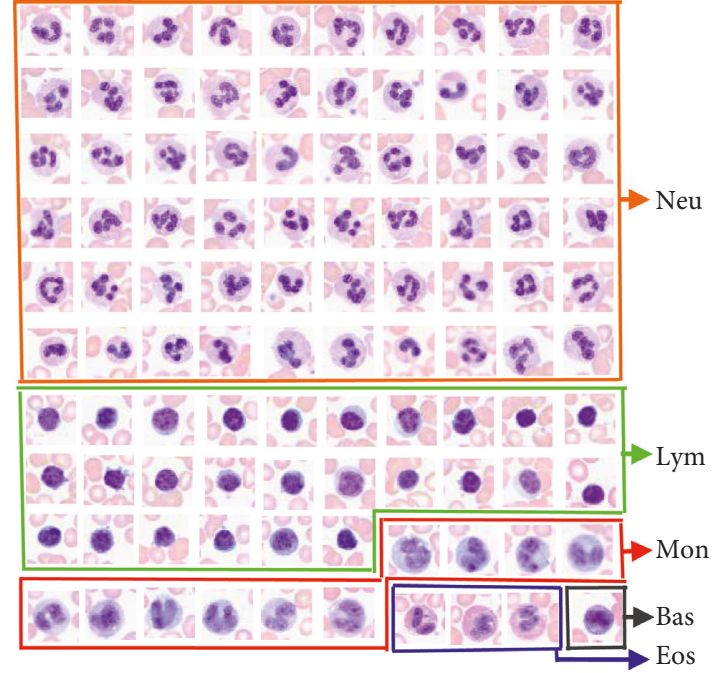

(b)

Figure 7: The leukocyte autoscanning test results for different blood smears at different conditions. (a) The extraction leukocyte images of blood smear sample 1. (b) The extraction leukocyte images of blood smear sample 2.

TABLE 3: The leukocyte counting results for ten tested samples (\%).

\begin{tabular}{lcccccccccc}
\hline \multirow{2}{*}{ Type } & \multicolumn{10}{c}{ Sample } \\
& 1 & 2 & 3 & 4 & 5 & 6 & 7 & 8 & 9 & 10 \\
\hline Neu & 66 & 60 & 62 & 65 & 55 & 60 & 50 & 66 & 52 & 42 \\
Lym & 24 & 26 & 24 & 25 & 35 & 30 & 35 & 25 & 35 & 26 \\
Mon & 5 & 10 & 8 & 7 & 8 & 7 & 6 & 6 & 8 & 20 \\
Eos & 4 & 3 & 5 & 3 & 2 & 2 & 8 & 3 & 4 & 8 \\
Bas & 1 & 1 & 1 & 0 & 0 & 1 & 1 & 0 & 1 & 4 \\
\hline
\end{tabular}

extracted leukocyte image, and thus it means that the proposed method can well meet the real-time application.

The experimental results show that the proposed segmentation method has good segmentation quality and quick speed; it has better stability and robustness.

3.4. Segmentation Results on Clumped Leukocytes. In order to verify the practicability of the proposed method on 


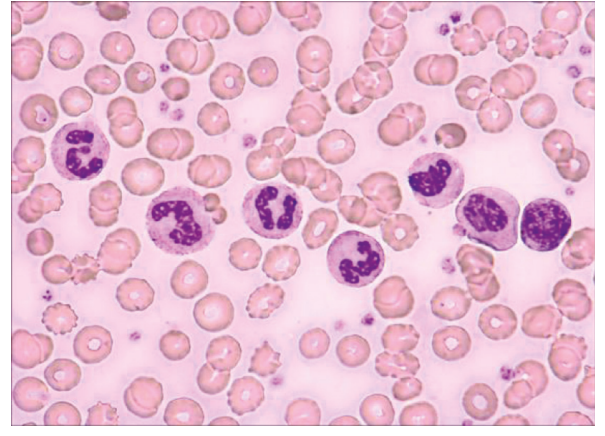

(a)

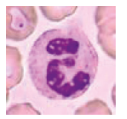

(c)

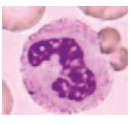

(d)

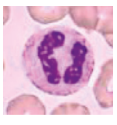

(e)

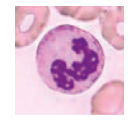

(f)

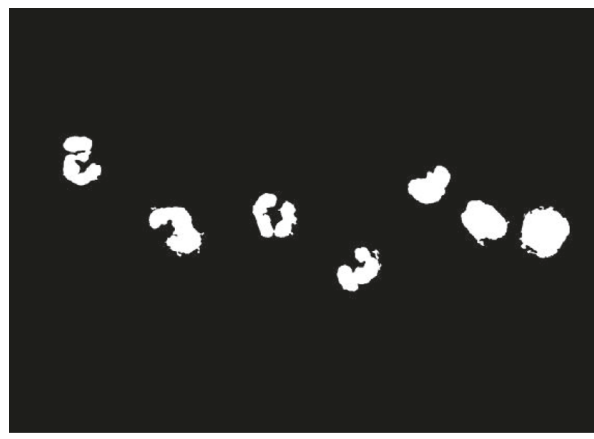

(b)

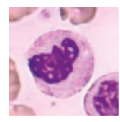

(g)

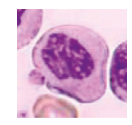

(h)

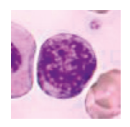

(i)

Figure 8: Clumped leukocytes and the segmentation result. (a) Clumped leukocytes. (b) Segmentation result. (c) Segmented image 1. (d) Segmented image 2. (e) Segmented image 3. (f) Segmented image 4. (g) Segmented image 5. (h) Segmented image 6. (i) Segmented image 7.

clumped human peripheral blood leukocytes, an experiment is conducted. As can be seen, the leukocytes are quite clumped in Figure 8(a). The segmentation result using our method is shown in Figure 8(b). The segmented images are Figures $8(\mathrm{c})-8(\mathrm{i})$. As can be seen, all the leukocyte nuclei can be segmented successfully. It indicates the practicability of the proposed method on clumped leukocytes. For a peripheral blood smear that meets the requirements, the overlap between leukocytes will not appear. We think that the proposed method will work well when there is no overlap between leukocytes in the image to be segmented.

\section{Conclusions}

A quick leukocyte nucleus segmentation method based on the $B-G$ values in leukocyte counting has been proposed. Because only an 8 bit subtraction operation is performed for $B-G$ values, it can reduce the amount of computation significantly. The average time consumption of the proposed method is only $0.26 \mathrm{~ms}$. Experimental results show its feasibility, robustness, and the better real-time performance. The proposed method can be applied successfully in the realtime autoscanning test for the leukocyte peripheral blood smear which includes autoscanning, autofocusing, autoextracting, and autocounting of leukocyte images to reduce the errors caused by manual operation. So, it guarantees sufficient results for doctor to diagnose the diseases more quickly and more effectively.

\section{Data Availability}

The image data used to support the findings of this study are available from the corresponding author upon request.

\section{Conflicts of Interest}

The authors declare that they have no conflicts of interest.

\section{Acknowledgments}

This work was supported by the 863 National Plan Foundation of China under grant no. 2007AA01Z333 and Special Grand National Project of China under grant no. 2009ZX02204-008.

\section{References}

[1] J. J. Castillo, N. Mull, J. L. Reagan, S. Nemr, and J. Mitri, "Increased incidence of non-Hodgkin lymphoma, leukemia, and myeloma in patients with diabetes mellitus type 2: a metaanalysis of observational studies," Blood, vol. 119, no. 21, pp. 4845-4850, 2012.

[2] L. Putzu, G. Caocci, and C. Di Ruberto, "Leucocyte classification for leukaemia detection using image processing techniques," Artificial Intelligence in Medicine, vol. 62, no. 3, pp. 179-191, 2014.

[3] S. Mohapatra, D. Patra, and S. Satpathy, "An ensemble classifier system for early diagnosis of acute lymphoblastic leukemia in blood microscopic images," Neural Computing and Applications, vol. 24, no. 7-8, pp. 1887-1904, 2014.

[4] Y. Liu, F. Cao, J. Zhao, and J. Chu, "Segmentation of white blood cells image using adaptive location and iteration," IEEE Journal of Biomedical and Health Informatics, vol. 21, no. 6, pp. 1644-1655, 2017.

[5] X. Li and Y. Cao, "A robust automatic leukocyte recognition method based on island-clustering texture," Journal of Innovative Optical Health Sciences, vol. 9, no. 1, article 1650009, 2016.

[6] H. Cao, H. Liu, and E. Song, "A novel algorithm for segmentation of leukocytes in peripheral blood," Biomedical Signal Processing and Control, vol. 45, pp. 10-21, 2018.

[7] K.-D. Hung, D.-C. Huang, and Y.-K. Chan, "A computer assisted method for leukocyte nucleus segmentation and recognition in blood smear images," Journal of Systems and Software, vol. 85, no. 9, pp. 2104-2118, 2012.

[8] N. I. C. Marzuki, N. H. Mahmood, and M. A. A. Razak, "Segmentation of white blood cell nucleus using active contour," Jurnal teknologi, vol. 74, no. 6, pp. 115-118, 2015.

[9] C. Pan, D. S. Park, S. Yoon, and J. C. Yang, "Leukocyte image segmentation using simulated visual attention," Expert Systems with Applications, vol. 39, no. 8, pp. 7479-7494, 2012. 
[10] M. Sezgin and B. Sankur, "Survey over image thresholding techniques and quantitative performance evaluation," Journal of Electronic Imaging, vol. 13, no. 1, pp. 146-165, 2004.

[11] Y. Yang, Y. Cao, and W. Shi, "A method of leukocyte segmentation based on S component and B component images," Journal of Innovative Optical Health Sciences, vol. 7, no. 1, pp. 1-8, 2014.

[12] N. Otsu, "A threshold selection method from gray-level histograms," IEEE Transactions on Systems, Man, and Cybernetics, vol. 9, no. 1, pp. 62-66, 1979.

[13] B. C. Ko, J.-W. Gim, and J.-Y. Nam, "Automatic white blood cell segmentation using stepwise merging rules and gradient vector flow snake," Micron, vol. 42, no. 7, pp. 695-705, 2011.

[14] F. Xing, Y. Xie, and L. Yang, "An automatic learning-based framework for robust nucleus segmentation," IEEE Transactions on Medical Imaging, vol. 35, no. 2, pp. 550-566, 2015.

[15] H. T. Madhloom, S. A. Kareem, H. Ariffin, A. A. Zaidan, H. O. Alanazi, and B. B. Zaidan, "An automated white blood cell nucleus localization and segmentation using image arithmetic and automatic threshold," Journal of Applied Sciences, vol. 10, no. 11, pp. 959-966, 2010.

[16] Y. Wen, Y. Lu, J. Yan, Z. Zhou, K. M. von Deneen, and P. Shi, "An algorithm for number plate recognition applied to intelligent transportation system," IEEE Transactions on Intelligent Transportation System, vol. 12, no. 3, pp. 830-845, 2011.

[17] Z.-X. Chen, C.-Y. Liu, F.-L. Chang, and G.-Y. Wang, "Automatic license-plate location and recognition based on feature salience," IEEE Transactions on Vehicular Technology, vol. 58, no. 7, pp. 3781-3785, 2009.

[18] A. Gautam, P. Singh, B. Raman et al., "Automatic classification of leukocytes using morphological features and naïve Bayes classifier," in Proceedings of the 2016 IEEE Region 10 Conference (TENCON), pp. 1023-1027, Singapore, November 2016.

[19] P. S. Hiremath, P. Bannigidad, and S. Geeta, "Automated identification and classification of white blood cells (leukocytes) in digital microscopic images," IJCA Special Issue on Recent Trends in Image Processing and Pattern Recognition (RTIPPR), vol. 2, 2010.

[20] S. H. Rezatofighi and H. Soltanian-Zadeh, "Automatic recognition of five types of white blood cells in peripheral blood," Computerized Medical Imaging and Graphics, vol. 35, no. 4, pp. 333-343, 2011.

[21] Y.-K. Chan, M.-H. Tsai, D.-C. Huang, Z.-H. Zheng, and K.-D. Hung, "Leukocyte nucleus segmentation and nucleus lobe counting," BMC Bioinformatics, vol. 11, no. 1, pp. 558-559, 2010. 


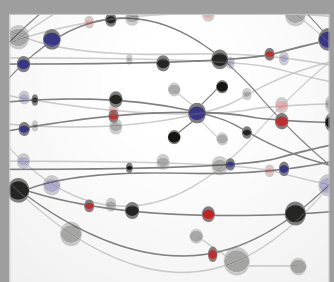

The Scientific World Journal
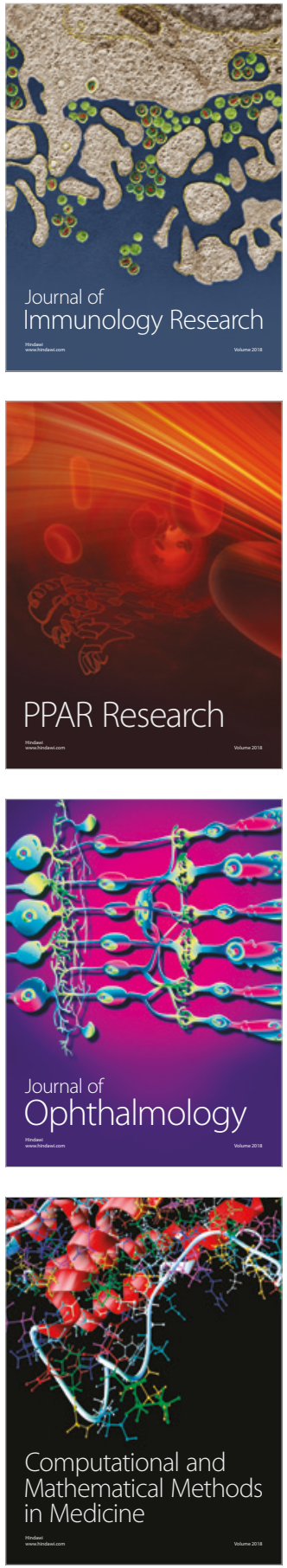

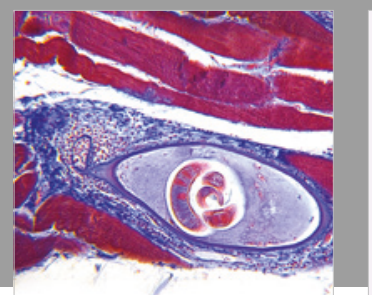

Gastroenterology Research and Practice

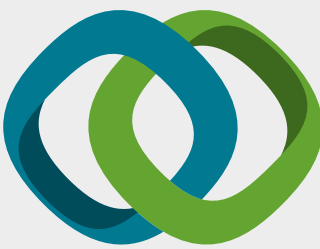

\section{Hindawi}

Submit your manuscripts at

www.hindawi.com
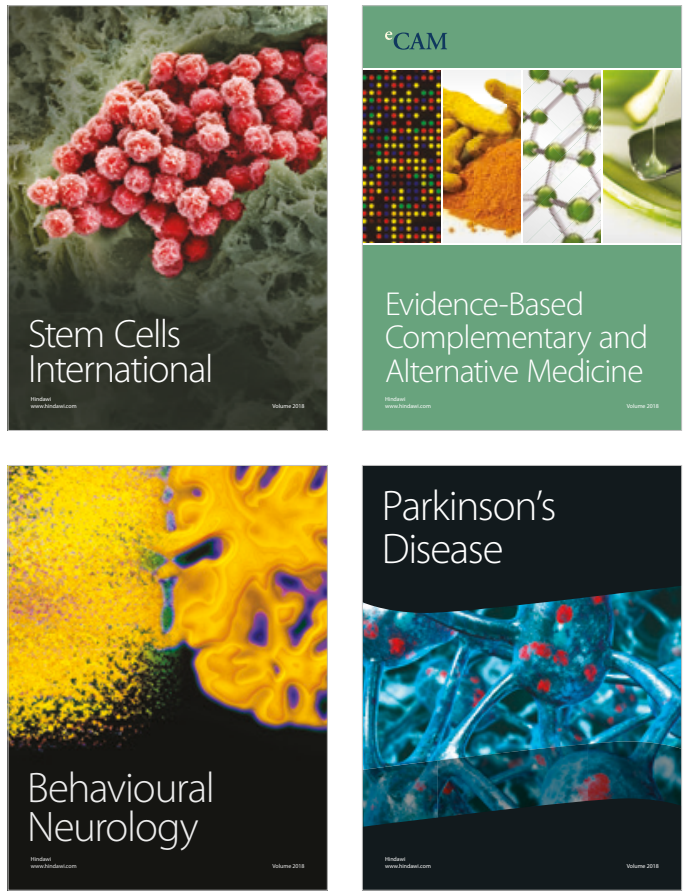

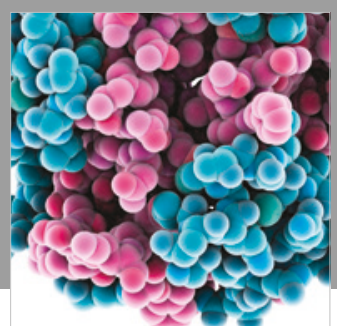

ournal of

Diabetes Research

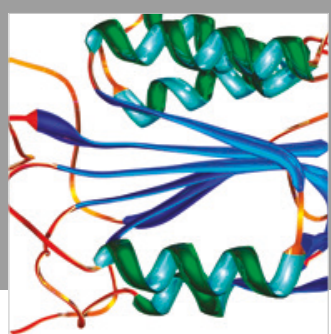

Disease Markers
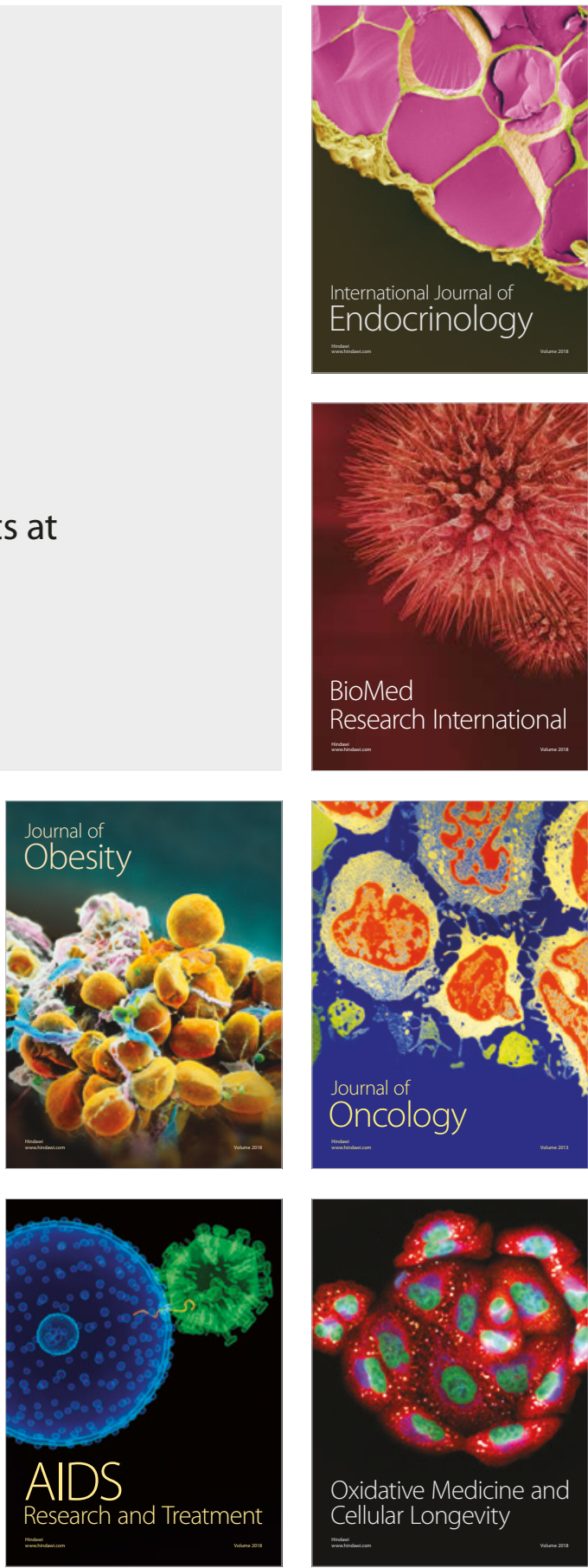